\title{
Deposição de serapilheira em uma área de caatinga preservada no semiárido da Paraíba, Brasil
}

\section{Litter deposition in a savanna area preserved in the semiarid region of Paraíba, Brazil}

\author{
Vinícius N. Silval ${ }^{1}$ Lauter S. Souto ${ }^{2}$, João de A. Dutra Filho ${ }^{3}$, Tarso M. A. de Souza ${ }^{4}$, César H. A. Borges ${ }^{5}$
}

\begin{abstract}
RESUMO: A quantificação da serapilheira depositada é de fundamental importância em estudos de ciclagem de nutrientes em áreas em processo de sucessão ecológica. Com esse fim, objetivou-se quantificar a deposição sazonal de serapilheira em um remanescente de caatinga em estágio avançado de regeneração no município de Cajazeirinhas, PB. As coletas foram realizadas mensalmente, durante o período de um ano, de agosto de 2013 a julho de 2014 em quatro transectos. Para isso, foram distribuídas 20 caixas coletoras de $1 \mathrm{~m} \times 1 \mathrm{~m}$, separando-se o material coletado nas seguintes frações: folhas, galhos, estruturas reprodutivas e miscelânea. A produção de serapilheira na área durante o período de estudo foi de $1630,5 \mathrm{~kg} \mathrm{ha}^{-1}$, sendo que na área a fração folhas predominou na serapilheira contribuindo ao solo com $64,9 \%$, seguida da fração galhos $(21,2 \%)$, estruturas reprodutivas $(10,1 \%)$ e miscelânea $(3,8 \%)$. A maior deposição ocorreu no início da estação seca, caracterizando a sazonalidade. Foi observado que depois do longo período de regeneração, a área apresenta padrão de deposição de serrapilheira similar ao registrado em outras áreas de caatinga preservada.
\end{abstract}

Palavras-chaves: Ciclagem biogeoquímica, Caatinga, Semiárido.

ABSTRACT: Quantification of litter produced is of fundamental importance in nutrient cycling studies in areas of ecological succession process. To this end, the objective was to quantify the seasonal deposition of litter in a remnant of caatinga in advanced stages of regeneration in the city of Cajazeirinhas, PB. Samples were collected monthly during the period of one year, from August 2013 to July 2014 in four transects. For this, were distributed twenty collection boxes of $1 \mathrm{~m} \times 1 \mathrm{~m}$, separating the material collected in the following fractions: leaves, branches, reproductive structures and miscellaneous. The litter production in the area during the study period was $1630,5 \mathrm{~kg} \mathrm{ha}^{-1}$, and the area fraction leaves predominated in burlap contributing to soil with $64,9 \%$, followed by fraction branches $(21,2 \%)$, reproductive structures $(10,1 \%)$ and miscellaneous $(3,8 \%)$. Most deposition occurred at the beginning of the dry season, featuring seasonality. It was observed that after the long period of regeneration, the area shows a pattern of litter deposition similar to that recorded in other areas of preserved savanna.

Key words: Biogeochemical cycling, Caatinga, Semiarid.

\footnotetext{
*Autor para correspondência

Recebido para publicação em 14/02/2015; aprovado em 22/04/2015

1 Graduando em Agronomia, UFCG, Campus Pombal-PB - Rua Jairo Vieira Feitosa, n. 1770, Bairro dos Pereiros, CEP: 58.840-000. Email:v11n1170@gmail.com

${ }^{2}$ Eng. Agrônomo, Dr. Agronomia, Professor UAGRA/CCTA/UFCG, Campus Pombal PB - Rua Jairo Vieira Feitosa, n. 1770, Bairro dos Pereiros, CEP: 58.840-000. Email: lautersouto@yahoo.com.br

${ }^{3}$ Biólogo, Dr. Genética, Professor UAGRA/CCTA/UFCG, Campus Pombal-PB - Rua Jairo Vieira Feitosa, n. 1770, Bairro dos Pereiros, CEP: 58.840-000. Email:filho-dutra@ig.com.br

${ }^{4}$ Graduando em Agronomia, UFCG, Campus Pombal-PB - Rua Coronel José Fernandes, n. 181, Centro, CEP: 58.840-000. Email:tarsobh@ hotmail.com

${ }^{5}$ Eng. Florestal, Mestrando em Ciências Florestais, UAEF/CSTR/UFCG, Campus Patos-PB - Av. Universitária, S/N, Bairro Santa Cecília, CEP: 58.708-110.

Email:cesarhenrique27@yahoo.com.br
} 


\section{INTRODUÇÃO}

A Caatinga é o bioma mais extenso da região Nordeste brasileiro, ocupando área de $844.453 \mathrm{~km}^{2}$ ou $11 \%$ do território nacional (MMA, 2014), possuindo significativa importância socioeconômica e ecológica. A caatinga engloba os estados de Alagoas, Ceará, Rio Grande do Norte, Paraíba e Pernambuco, Piauí, Sergipe, Bahia e uma faixa seguindo o rio São Francisco em Minas Gerais (Prado, 2003). É um bioma caracterizado por um déficit hídrico acentuado, altas taxas de insolação e evapotranspiração, além de reduzida e variável precipitação, que acaba determinando a distribuição das espécies e a produção de biomassa.

Apesar da significativa extensão e importância deste bioma, a caatinga é o menos protegido dentre os biomas brasileiros, estando menos de $2 \%$ de sua área protegida sob a forma de unidades de conservação de proteção integral (Tabarelli et al., 2000). Além disso, o impacto da atividade humana sobre o bioma é descontrolado, danoso e considerável, aumentando os níveis de degradação do bioma de forma acentuada.

Estudos realizados em ecossistemas naturais são de relevante importância para o entendimento do comportamento das características intrínsecas dos ecossistemas. Neste contexto, deve-se levar em consideração a dinâmica nutricional do bioma em relação à ciclagem biogeoquímica, que ocorre naturalmente, pela lavagem das copas e troncos das árvores pelas chuvas ocasionadas ao longo do ano, ao atravessar o dossel das espécies vegetais e através da deposição de tecidos senescentes logo após a sua decomposição (Balieiro et al. (2004).

Desta forma, o processo de deposição da serapilheira, incluindo as taxas anuais de queda do material decíduo e o processo de decomposição desse material, devem ser amplamente estudados, especialmente em condições semiáridas, onde há grande ocorrência de solos com baixos níveis de nutrientes por ser um fator chave na manutenção dos nutrientes no ecossistema.

São poucos os conhecimentos sobre os ecossistemas naturais e sobre a ciclagem de nutrientes em florestas naturais no Brasil. Portanto, torna-se necessário o desenvolvimento de novas pesquisas, principalmente naquelas regiões do país mais sujeitas aos impactos antrópicos, onde os ecossistemas primitivos se encontram em via de desaparecimento (Caldeira et al., 2010).

Ao comparar o bioma caatinga com outras regiões semiáridas do mundo, percebemos que ela também é a mais rica em biodiversidade, apresentando alto grau de espécies únicas. Portanto, a nossa Caatinga é exclusivamente brasileira e grande parte de suas espécies não são encontradas em nenhuma outra parte do planeta. Isso significa que a nossa responsabilidade de cuidar desse patrimônio é grande, que devemos ser especialmente comprometidos com ele, pois existem poucos remanescentes de Caatinga preservados e muitas de suas espécies estão ameaçadas de extinção.

Este trabalho teve como objetivo quantificar a deposição de serapilheira em um remanescente de caatinga no município de Cajazeirinhas, $\mathrm{PB}$, durante o período de agosto de 2013 a julho de 2014.

\section{MATERIAL E MÉTODOS}

O experimento foi instalado na Fazenda São Francisco, situada no município de Cajazeirinhas, $\mathrm{PB}$, latitude $06^{\circ}$ $57^{\prime} 40^{\prime \prime} \mathrm{S}$ e longitude $37^{\circ} 48^{\prime} 22^{\prime} \mathrm{W}$. O fragmento possui uma área de 30 ha que não é explorada há mais de 30 anos. Os solos predominantes da área experimental são LUVISSOLOS em associação com NEOSSOLOS LITÓLICOS (EMBRAPA, 2013).

O clima da região segundo a classificação de Köeppen se enquadra no tipo BSh, semiárido quente, com médias térmicas anuais superiores a $25^{\circ} \mathrm{C}$ e pluviosidade média anual inferior a $1000 \mathrm{~mm} / \mathrm{ano}$ com chuvas irregulares.

$\mathrm{Na}$ área foram demarcados quatro transectos distantes $50 \mathrm{~m}$ entre eles. Neles foram instalados coletores distando $25 \mathrm{~m}$ entre si, totalizando 20 caixas coletoras. O acompanhamento da produção de serapilheira compreendeu o período de agosto de 2013 a julho de 2014. Os coletores (Figura 1) apresentavam 1,0m x 1,0m, com os lados formados de madeira com altura de $15,0 \mathrm{~cm}$ e o fundo de tela de náilon com malha de $1,0 \mathrm{~mm} \times 1,0 \mathrm{~mm}$, suspensos a $15 \mathrm{~cm}$ acima da superfície do solo.

Mensalmente as amostras coletadas foram submetidas à secagem a $65^{\circ} \mathrm{C}$ até peso constante. Após este procedimento, o material foi encaminhado ao Laboratório de Fitotecnia do CCTA/UFCG, para separação dos constituintes (Figura 1): folhas, estruturas reprodutivas (flores, frutos e sementes), galhos (correspondente às partes lenhosas arbóreas de todas as dimensões e cascas) e miscelânea (material vegetal que não pode ser determinado e material de origem animal).

A produção de serapilheira foi estimada através da seguinte equação: PAS $=(\mathrm{PS} \times 10.000) / \mathrm{Ac}$, em que: $\mathrm{PAS}=$ produção média anual de serapilheira $\left(\mathrm{kg} \mathrm{ha}^{-1}\right.$ ano); PS = produção média mensal de serapilheira $\left(\mathrm{kg} \mathrm{ha}^{-1} \mathrm{mês}^{-1}\right)$; e Ac = área do coletor $\left(\mathrm{m}^{2}\right)$.
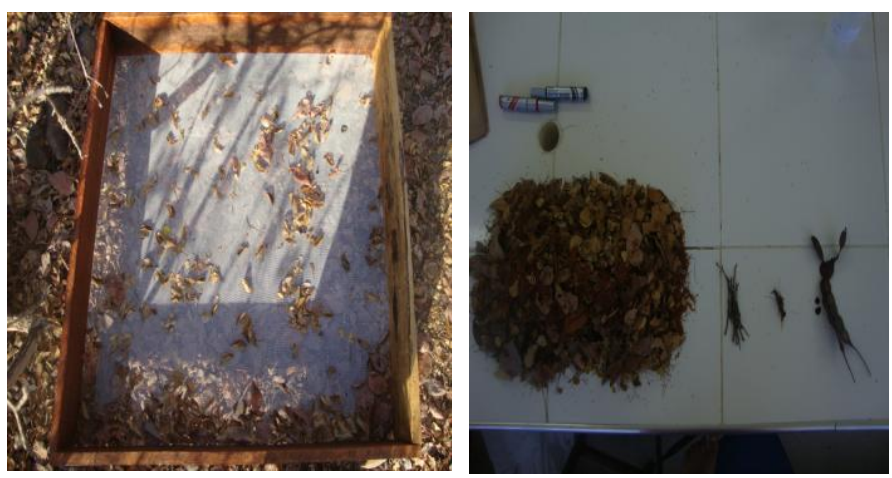

Figura 1. Caixa coletora utilizadas nos estudos de deposição (a) e separação das frações folhas, galhos, estruturas reprodutivas e miscelânea (b). Pombal, PB. 2014.

\section{RESULTADOS E DISCUSSÃO}

A precipitação total entre o período de janeiro de 2013 a junho de 2014 foi de 1455,9mm (Figura 2). Durante esse período foi observado que as maiores precipitações ocorreram nos primeiros seis meses de cada ano, com totais de $509,0 \mathrm{~mm}(76,6 \%)$ e $751,3 \mathrm{~mm} \quad(95,0 \%)$ do total precipitado, respectivamente. Período este que antecedeu as maiores deposições das frações da serapilheira (Tabela 2). 
Nos demais meses do ano (2013 e 2014) as taxas de precipitação caracterizam o regime de distribuição irregular das chuvas de regiões semiáridas e a respectiva perda de folhas características das espécies deste bioma.

A análise final dos dados da serapilheira mostrou diferenças entre as médias de deposição por frações. A quantidade de folha coletada nesse período foi superior à quantidade de galhos, e esta foi superior à quantidade de estrutura reprodutiva e miscelânea. Estes valores correspondem respectivamente a $64,9 \%\left(1058,19 \mathrm{Kg}^{-h^{-1}}\right)$; 21,2\% (345,67 Kg.ha $\left.{ }^{-1}\right) ; 10,1 \%$ (164,68 Kg.ha $\left.{ }^{-1}\right)$; e 3,8\% $\left(61,96 \mathrm{Kg} \mathrm{ha}^{-1}\right)$. Com isso, observou-se que durante esse período de 10 meses obteve-se um total de serapilheira produzida de $1630,5 \mathrm{~kg} \cdot \mathrm{ha}^{-1}$ depositada sobre a superfície do solo. Segundo Delitti (1995), o total de serapilheira depositada no solo pode variar entre os diferentes ecossistemas.

Devido ao período de estiagem, a escassez de água fez com que as plantas perdessem as folhas, como uma medida preventiva para evitar a perda de água por transpiração. Desta forma, as folhas é a principal fração que contribui com o acúmulo de serrapilheira sobre a superfície do solo, configurando uma eficiente camada protetora contra o impacto das gotas de chuva (splash) sobre a superfície do solo, diminuindo assim, os riscos de erosão dos solos pelo processo erosivo. Vários pesquisadores como Souto (2006), Santana (2005), Alves et al. (2006), Lopes et al. (2009) e Paula et al. (2009) reportam em seus trabalhos que a fração folha contribui com as maiores taxas de deposição.

$\mathrm{Na}$ tabela 2 , pode-se observar a variação mensal da produção de materiais orgânicos nas diferentes frações. Podese observar que na fração folha verificou-se diferenças na taxa de deposição durante o período de avaliação e que foi no mês de junho na fração folha que, obteve-se a maior taxa de deposição com o valor de 373,0 Kg.ha ${ }^{-1}$. Quando comparado aos demais meses, esse valor corresponde a $35,24 \%$ do total de serapilheira produzida durante os 10 meses avaliados. De um modo geral, observou-se que entre os meses de dezembro de 2013 à março de 2014 obteve-se a menores valores de deposição da fração folha.

Durante o período de 10 meses, a fração estrutura reprodutiva teve em média uma deposição de 16,46 Kg.ha ${ }^{-1}$, estando o mês de fevereiro com a maior taxa de aporte desta fração com 41,0 Kg.ha ${ }^{-1}$ o que corresponde a $24,89 \%$ no total. A fração galho no período avaliado de Setembro de 2013 a Junho de 2014 apresentou grande variação na deposição ao longo deste período, e teve um valor médio de material depositada ao solo de $34,56 \mathrm{Kg} \mathrm{ha}^{-1}$ e tendo o mês de março como o de menor taxa de deposição com 18,6 Kg.ha ${ }^{-1}$.

Observou-se que a fração miscelânea obteve um valor muito maior no mês de junho quando comparado com os meses anteriores, apresentando uma taxa no valor de 19,1 Kg.ha ${ }^{-1}$, correspondendo a $30,82 \%$ no valor total do período avaliado, isso se deve ao fato de apresentar varias estruturas minúsculas depositadas e assim prejudicando o reconhecimento da mesma.

A fração folha foi a que apresentou a maior taxa de deposição, com 1.058,2 Kg.ha' ${ }^{-1}$. Os meses de janeiro, fevereiro e abril tiveram maiores precipitações pluviométricos com 160,2 mm, 201,0 mm e 170,3 mm, respectivamente, e consequentemente pouca produção de serapilheira, já no mês de junho que ouve estiagem, obteve-se o maior numero de produção da fração.

A análise sazonal das variações na deposição da serapilheira no Bioma Caatinga permite que se tenha o conhecimento de como as diferentes espécies respondem às características físicas e químicas do meio, a distribuição e estoque dos nutrientes, além de entender as estratégias usadas pela vegetação na manutenção da sustentabilidade do ecossistema (Souto, 2006).

Vários fatores afetam a quantidade de resíduos que caem da parte aérea das plantas que irão formar a serapilheira, com destaque para o clima, o solo, as características genéticas das plantas, a idade e a densidade de plantas, e de um modo geral, a distribuição das chuvas ao longo do tempo a qual influencia a disponibilidade de água no solo e determina a produtividade vegetal (Correia e Andrade, 1999).

$\mathrm{Na}$ produção de estruturas reprodutivas, observou-se que os meses de fevereiro e março tiveram o valor maior de serapilheira com 41,0 e 29,6 Kg.ha ${ }^{-1}$ respectivamente, e tendo dezembro, janeiro e junho como os meses de menores valores com 5,2, 8,1 e 2,6 Kg.ha ${ }^{-1}$, respectivamente.

É de grande importância acompanhar a deposição da fração estrutura reprodutiva, pois permite conhecer o comportamento fonológico das espécies, e de certa forma dar embasamento para estudos relacionados com o tema. Em seu estudo sobre a deposição de serapilheira em uma área de caatinga no município de Santa Terezinha, PB, Andrade et al. (2008) verificou que a fração estrutura reprodutiva, que inclui flores, frutos e sementes foi responsável por $8,72 \%$ da produção total da serrapilheira, o que correspondeu a 198,96 kg.ha ${ }^{-1}$.

A maior contribuição da fração galho depositada ao solo foi observada nos meses de novembro/2013 e junho/2014 com taxa de deposição de 63,4 e $65,5 \mathrm{Kg} \mathrm{ha}^{-1}$, respectivamente, assim totalizando $126,90 \mathrm{Kg} \cdot \mathrm{ha}^{-1}$, ou seja, $36,71 \%$ da fração galho que apresentou $345,67 \mathrm{Kg} \cdot \mathrm{ha}^{-1}$ sendo a segunda maior contribuição de serapilheira. Verificou-se que nos meses de março e maio ocorreram as menores taxas de deposição de galhos, com 18,6 e 18,7 Kg.ha ${ }^{-1}$, respectivamente. Essa fração, apesar de contribuir com expressiva biomassa da serapilheira em todos os biomas, é pouco estudada e compreendida, apresentando resultados extremamente variáveis, possivelmente em função da metodologia de coleta utilizada, como o diâmetro mínimo dos galhos e a área dos coletores (Santana, 2005).

A comparação dos dados com a fração galhos é dificultada porque, ao contrário da fração folhas que são amostradas por métodos semelhantes nos mais variados ecossistemas, não existe padronização quanto às dimensões (Souto, 2006).

Para a fração miscelânea, obteve-se uma deposição total de $61,96 \mathrm{Kg} \cdot \mathrm{ha}^{-1}$, sendo o maior pico registrado no mês de junho com $19,1 \mathrm{Kg} \cdot \mathrm{ha}^{-1}$,ou seja, $30,82 \%$ no valor total da fração (Tabela 2). Nesta fração, houve uma maior dificuldade em sua identificação, pois contém os próprios resíduos vegetais bastante fragmentados, parte de insetos, fezes e outros materiais de difícil reconhecimento. 


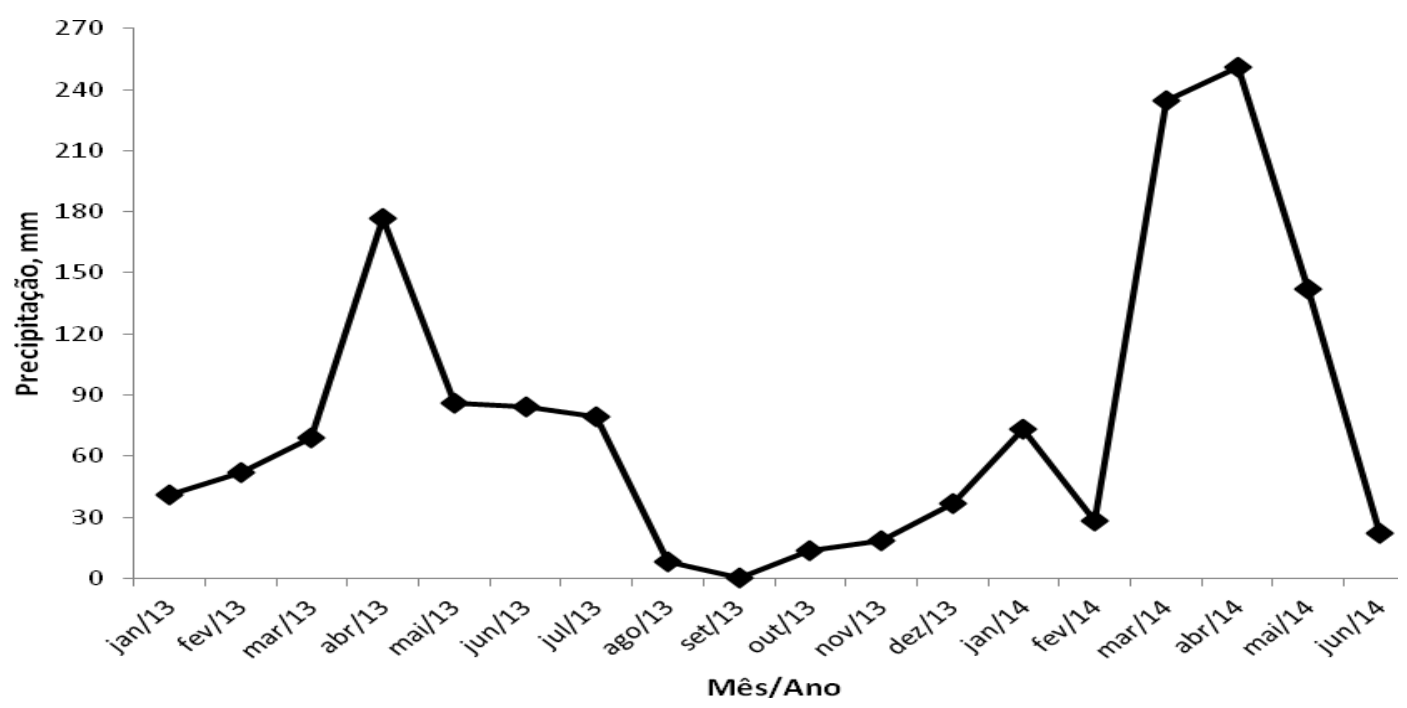

Figura 2. Precipitação pluviométrica registrada na área de estudo entre o período de Janeiro de 2013 a Junho de 2014. Cajazeirinhas, PB. 2014.

Tabela 1. Produção total $\left(\mathrm{Kg}_{\mathrm{g}} \mathrm{ha}^{-1}\right)$ e percentual $(\%)$ da serapilheira durante 10 meses em um remanescente de Caatinga localizado no município de Cajazeirinhas, PB. 2014.

\begin{tabular}{lcc}
\hline Frações & Produção Total $\left(K_{\text {Kg.ha }} \mathbf{- 1}^{\mathbf{1}}\right)$ & Percentual $(\boldsymbol{\%})$ \\
\hline Folha & $1.058,19$ & 64,9 \\
Galho & 345,67 & 21,2 \\
E. Reprodutiva & 164,68 & 10,1 \\
Miscelânea & 61,96 & 3,8 \\
\hline Total & $\mathbf{1 . 6 3 0 , 5}$ & $\mathbf{1 0 0}$ \\
\hline
\end{tabular}

Tabela 2. Médias mensais de deposição das frações folha, galhos, estrutura reprodutiva e miscelânea em Kg.ha ${ }^{-1}$. Cajazeirinhas, PB, 2014.

\begin{tabular}{lcccc}
\hline Meses & Folha & E. Reprodutiva & Galho & Miscelânea \\
\hline Setembro/2013 & & $-14,6$ & 38,7 & 2,1 \\
Outubro/2013 & 141,2 & 14,3 & 32,8 & 1,4 \\
Novembro/2013 & 62,3 & 18,3 & 63,4 & 1,7 \\
Dezembro/2013 & 87,1 & 5,2 & 21,4 & 4,6 \\
Janeiro/2014 & 34,4 & 8,1 & 34,1 & 2,2 \\
Fevereiro/2014 & 43,4 & 41,0 & 23,9 & 4,2 \\
Março/2014 & 74,0 & 29,6 & 18,6 & 8,6 \\
Abril/2014 & 40,1 & 12,0 & 23,5 & 14,7 \\
Maio/2014 & 90,5 & 8,0 & 18,7 & 3,3 \\
Junho/2014 & 112,1 & 2,6 & 65,4 & 19,1
\end{tabular}

Os maiores valores de deposição de serapilheira em regiões semiáridas vêm sendo registrados entre o período de transição das estações chuvosa e seca (Costa et al., 2007), porque nesse período os totais pluviométricos tornam-se reduzidos. Em resposta a baixa disponibilidade hídrica da estação seca, as plantas perdem a folhagem, favorecendo o aumento da deposição da serapilheira (Araújo et al., 2007).

\section{CONCLUSÕES}

A deposição de serapilheira observada na área de estudo demonstra a importância desta via de ciclagem de nutrientes para manutenção da produtividade do bioma caatinga, que é altamente dependente da diversidade de espécies presentes;

A fração folhas apresentou-se como predominante na serapilheira devolvida ao solo; 
A deposição e acúmulo da serapilheira variaram durante os meses avaliados em função da distribuição das chuvas, pois, interfere nos mecanismos fisiológicos das espécies da caatinga.

\section{REFERÊNCIAS BIBLIOGRÁFICAS}

ALVES, A. R. et al. Aporte e deposição em área de caatinga, na Paraíba. Revista de Biologia e Ciências da Terra Campina Grande, v. 6, n. 2, p. 194-203, 2006.

ANDRADE, A. G. et al. Deposição e decomposição da serapilheira em povoamentos de Mimosa caesalpiniiflolia, Acácia mangium e Acácia holosericea com quatro anos de idade em Planossolo. Revista brasileira de Ciência do Solo, Campinas, SP, v. 24, p. 777-785, 2000.

ARAUJO, E. L. et al. Dynamics of Brazilian Caatinga - A review concerning the plants, environment and people. Functional Ecology and Communities, v. 1, n.1, p. 15$28,2007$.

BALIEIRO, F. C. et al. Dinâmica de serapilheira e transferência de nitrogênio ao solo em plantios de Pseudosamanea guachapele and Eucalyptus grandis. Pesquisa Agropecuária Brasileira, Brasília, DF, v.39, n.6, p.597-601, 2004.

CALDEIRA, M.V.W. et al. Ciclagem de nutrientes, via deposição e acúmulo de serapilheira, em ecossistemas florestais. In: CHICHORRO, J. F.; GARCIA, G. O.; 15 BAUER, M. O.; CALDEIRA, M. V. W. Tópicos em ciências florestais. Visconde do Rio Branco: Suprema, 2010. Cap. 2, p.57-82.

COSTA, C. C. A. et al. Produção de serapilheira na caatinga da floresta nacional do Açú-RN. Revista Brasileira de Biociências, Porto Alegre, RS, v. 5, Supl.1, p. 246-248, 2007.

COSTA, G. S. et al. Aporte de nutrientes pela serapilheira em uma área degradada e revegetada com leguminosas arbóreas. Revista Brasileira de Ciência do Solo, v. 28, n.5, p. 919-927, 2004

DELITTI, W. B. C. Estudos de ciclagem de nutrientes: instrumentos para análise funcional de ecossistemas terrestres. Oecologia Brasiliensis, Rio de Janeiro, RJ, v. 1, n. 1, p. 469-483, 1995.

EMBRAPA. Sistema Brasileiro de classificação de solos. Brasília: EMBRAPA - Produção de Informação. Rio de Janeiro: Embrapa Solos, 2013. 353p.

MMA, 2014. Caatinga. Disponível em: http://www.mma.gov.br/biomas/caatinga. Acesso em 10 de novembro de 2014 .

LOPES, J. F. B. et al. Deposição e decomposicao de serrapilheira em area de caatinga. Revista Agroambiente On-line, Boa Vista, v. 3, n. 2, p.72-79, 2009.

PAULA, R. R. et al. Aporte de nutrientes e decomposição da serapilheira em três fragmentos florestais periodicamente inundados na ilha da Marambaia, RJ. Ciência Florestal, Santa Maria, v. 19, n. 2, p. 139-148, abr./jun., 2009.

PRADO, D. E. As caatingas da América do Sul. In: LEAL, I.R.; TABARELLI, M.; SILVA, J.M.C. (Eds.) Ecologia e conservação da Caatinga. Recife: Editora Universitária da UFPE. 2003. p.3-74.

SANTANA, J. A. da S. Estrutura fitossociológica, produção de serapilheira e ciclagem de nutrientes em uma área de Caatinga no Seridó do Rio Grande do Norte. 2005. 184 f. Tese (Doutorado em Agronomia) Centro de Ciências Agrárias, Universidade Federal da Paraíba, Areia, PB.

SOUTO, P. C. Acumulação e decomposição de serapilheira e distribuição de organismos edáficos em área de Caatinga na Paraíba, Brasil. 2006. 150p. Tese (Doutorado em Agronomia) - Centro de Ciências Agrárias, Universidade Federal da Paraíba, Areia - PB. 2006.

TABARELLI, M. et al. Análise de representatividade das unidades de conservação de uso direto e indireto na Caatinga: análise preliminar. In: Avaliação e identificação de ações prioritárias para a conservação, utilização sustentável e repartição de benefícios da biodiversidade do bioma Caatinga. Petrolina, Pernambuco. 2000. 